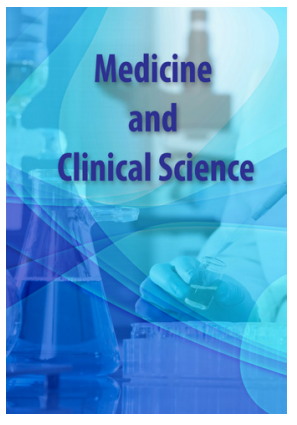

Correspondence

Yuriko Matsumura

Division of Infection Prevention and Control Tokyo Healthcare University, 4-1-17 HigashiGotanda, Shinagawa-ku, Tokyo 141-8648, Japan

Tel: 81-3-5421-7685

E-mail: y-matsumura@thcu.ac.jp

- Received Date: 21 Nov 2019;

- Accepted Date: 03 Dec 2019;

- Publication Date: 06 Dec 2019.

Keywords:

influenza virus, virucidal activity, tropolone derivatives

Copyright

(c) 2019 Science Excel. This is an openaccess article distributed under the term of the Creative Commons Attribution 4.0 International license.

\title{
Synthesis and virucidal activity of 5-substituted tropolones
}

\author{
Yuriko Matsumura ${ }^{1,2^{*}}$, Hirotaka Kato ${ }^{2}$, and Atsuo Iwasawa ${ }^{1}$ \\ ${ }^{1}$ Division of Infection Prevention and Control, Tokyo Healthcare University, Japan \\ ${ }^{2}$ Faculty of Science and Technology, Seikei University, Japan
}

\begin{abstract}
In 2009, a new type of influenza A virus (H1N1) led to an influenza pandemic. This particular virus subsequently acquired drug resistance, and the development of new virucidal agents is therefore urgently required to overcome the threat of further infection in the future. Hinokitiol and its derivatives have been reported to exhibit a broad range of anti-bacterial and anti-fungal activities. Nine different 5-substituted tropolones have been synthesized in the current study and evaluated in terms of their virucidal activity against five kinds of influenza virus. Only 5-substituted tropolones bearing nitro and iodo groups showed virucidal activity. These results suggested that tropolone derivatives of this type required a low pKa value and hydrophilic properties to show virucidal activity.
\end{abstract}

\section{Introduction}

Virucidal and anti-fungal agents are essential for the treatment of infections. Influenza is one of the most highly contagious forms of acute respiratory infection, and a new type of influenza A virus (H1N1) led to an influenza pandemic in 2009 [1]. This particular virus has subsequently acquired drug resistance and there is thus an urgent need for the development of new virucidal agents for the treatment of infections resulting from this virus.

Hinokitiol ( $\beta$-thujaplicin: Figure 1 ) is a component of essential oils, which was first isolated from Chamaecyparis taiwanensis by Nozoe in 1936 [2].

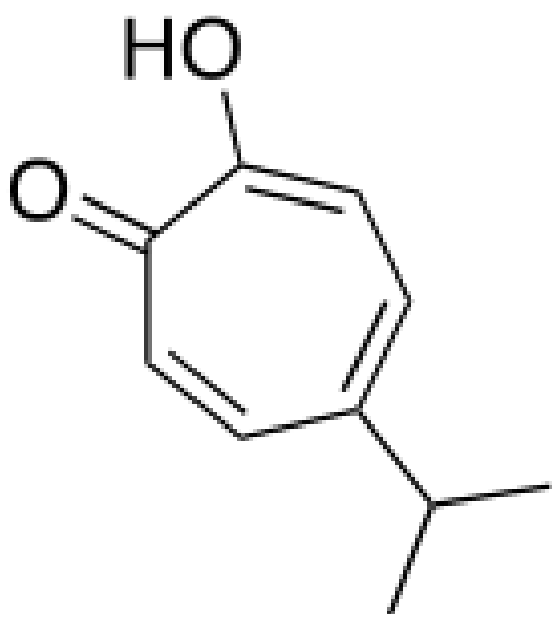

Figure 1. Structure of hinokitiol
In terms of its chemical structure, hinokitiol is a seven-membered tropolone bearing an isopropyl group at its 5-position. Compounds belonging to this structural class have been reported to exhibit a broad range of interesting biological properties, including anti-bacterial, anti-fungal, and anticancer activities [3-6]. Furthermore, hinokitiol can be found in a number of commercial health care products, including toothpaste, cosmetics, oral care gel, skin lotion and body soap, because of its wide range of biological properties [5]. The application of hinokitiol, however, has been limited by its poor aqueous solubility. The poor solubility of hinokitiol has been attributed in part to the isopropyl group at the C-5 position of its tropolone skeleton, which is responsible for the hydrophobic nature of the compound. To further evaluate the antimicrobial activity of hinokitiol derivatives, it would be useful to improve their solubility properties. In 2007, Morita [7] suggested that the free hydroxyl group at the C-2 position of the tropolone skeleton was critical to the bactericidal activity of hinokitiol-based compounds. Furthermore, the $\mathrm{pKa}$ value of this hydroxyl group can be affected significantly by the nature of the functional group at the C-5 position. Although numerous studies have been reported pertaining to the synthesis of tropolone derivatives, studies focused on the virucidal activities of compounds of this type are scarce.

Herein, we report the synthesis of the nine different 5 -substituted tropolones and an investigation of the effect of the $\mathrm{pKa}$ of the hydroxyl group at their C-2 position on their virucidal activity against five types of influenza virus.

\section{Materials and Methods}

\section{Synthesis of 5-substituted tolopolones}

The route used to synthesized the 5-substituted tropolones is summarized in Scheme 1. The detail information is available on the supplementary data.

Citation: Matsumura Y. Synthesis and virucidal activity of 5-substituted tropolones. Med Clin Sci. 2019;1(2):1-4. 


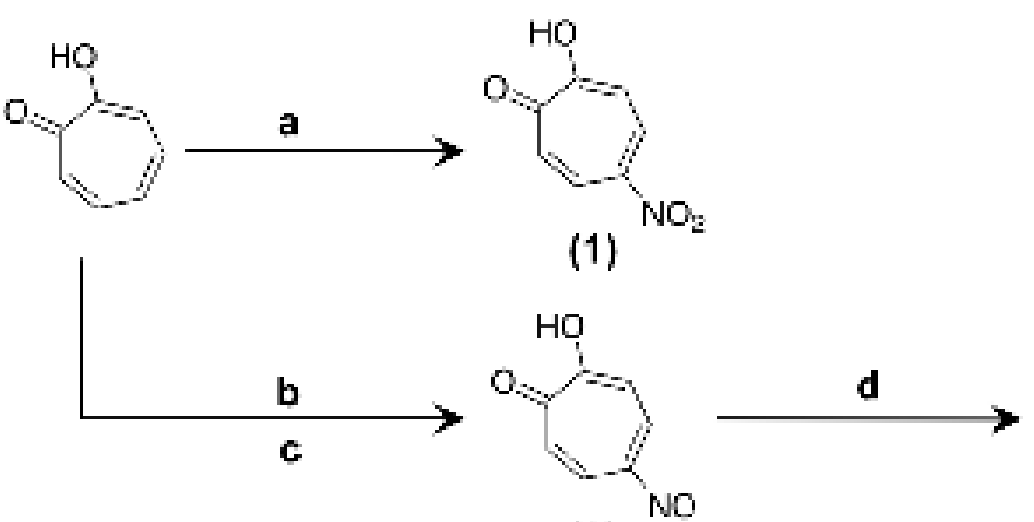

(2)

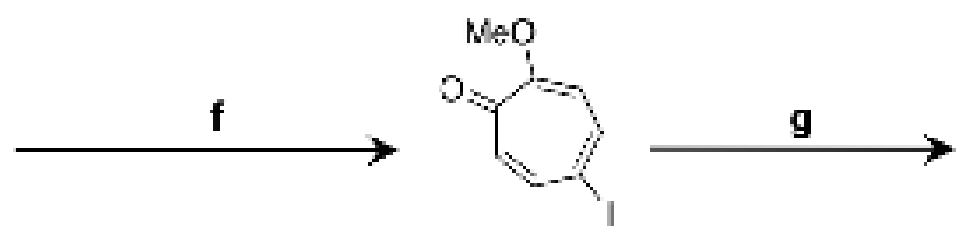

(5)

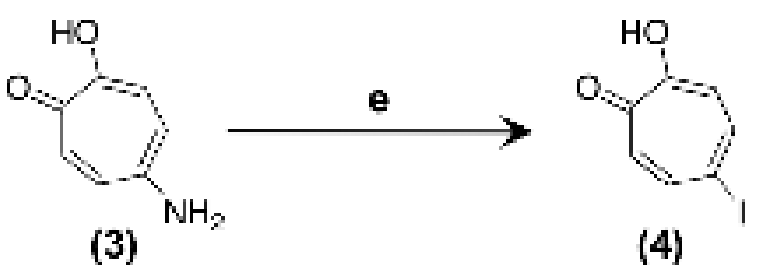

(3)

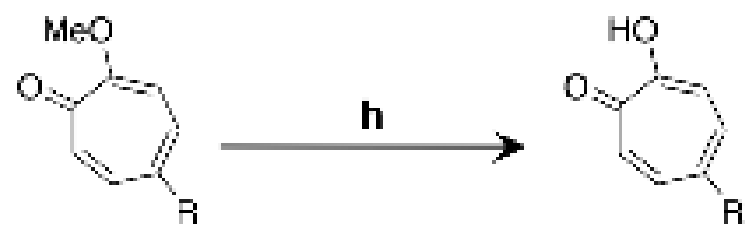

(6-8)

(6), (9) $\mathbf{R}=$ phenyl

(7), (10) $\mathrm{R}=p-\mathrm{CH}_{3}$-phenyl

(8), (11) $\mathrm{R}=$ thienyl

Scheme 1. Reagents and conditions: (a) $\mathrm{HNO}_{3^{\prime}} \mathrm{H}_{2} \mathrm{O}$; (b) $\mathrm{NaNO}_{2^{\prime}} \mathrm{H}_{2} \mathrm{O}$, glacial AcOH; (c) $6 \mathrm{M} \mathrm{HCl}$; (d) $\mathrm{H}_{2^{\prime}}, 10 \% \mathrm{Pd} / \mathrm{C}, \mathrm{MeOH}$; (e) $\mathrm{NaNO}{ }^{\prime}, \mathrm{HCl}, \mathrm{Kl}, \mathrm{H}_{2} \mathrm{O}$; (f) $(\mathrm{Me})_{2} \mathrm{SO}_{4^{\prime}} \mathrm{K}_{2} \mathrm{CO}_{3^{\prime}}$ acetone, $\mathrm{H}_{2} \mathrm{O}$; (g) $(\mathrm{OH})_{2} \mathrm{~B}-\mathrm{R},\left(\mathrm{PPh}_{3}\right)_{4} \mathrm{Pd}, 2 \mathrm{M} \mathrm{Na}_{2} \mathrm{CO}_{3^{\prime}}$ (h) $1 \mathrm{M} \mathrm{BBr}_{3^{\prime}} \mathrm{CH}_{2} \mathrm{Cl}_{2}$.

\section{Measurement of $\mathrm{pKa}$ values}

The $p K a$ values were measured by the $\mathrm{pH}$ titration method or the UV titration method. Potassium hydroxide solution was used as the alkaline solution.

\section{Measurement of the partition coefficient}

The partition coefficients were measured by using an PBS buffer solution at $\mathrm{pH} 7.4$ and 1-octanol. To a solution of the compound in 1-octanol (5 $\mathrm{mL})$ was added PBS buffer solution $(5 \mathrm{~mL})$. The mixture was stirred at $600 \mathrm{rpm}$ for $4 \mathrm{~h}$ with a magnetic stirrer, and then centrifuged at 4000 rpm for 20 min with a centrifugal separator. The concentrations of two solutions were determined by measuring UV-Vis spectra. The partition coefficient was calculated from the following equation;

$\log$ Pow $=\mathrm{C}_{\text {octanol }} / \mathrm{C}_{\text {buffer }}$.

\section{Virucidal activity}

The four kinds of influenza virus, A/PR8, A/USSR/92/97/ A/FM/1/47, and A.pdm.2009No.18, and MDCK cell were used for the evaluation of the virucidal activity. One hundred $\mu \mathrm{L}$ of viral preparation was added to $900 \mu \mathrm{L}$ of test solution containing 5-substituted tropolone dissolved in DMSO $(1 \mathrm{mM})$, and the viral quantification assay was performed after 1 and 5 min. DMSO served as a control. The mixture of the viral preparation and test solution was dispended to each well with corresponding susceptible cells in a 96-well microplate. The concentration of FBS in the viral preparation was kept at $1 \%$ during viral culture. After 1 hour incubation for infection at $37^{\circ} \mathrm{C}, 100$ $\mu \mathrm{L}$ of medium was added and further incubated in $5 \% \mathrm{CO}_{2}$ until quantification. Virucidal activity was determined after four to six days.

\section{Results and discussion}

\section{Synthesis and chemical properties of 5-substituted tropolones}

5-Nitorotropolone (1) was synthesized by the nitration of tropolone according to the method reported by Adachi [8]. All of the other 5 -substited tropolones were synthesized via a nitrosation reaction [9-10]. Briefly, catalytic reduction of the resulting nitroso group gave aniline 3, which was converted to the corresponding iodide 4 using a Sandmeyer reaction [9]. Compound 4 was converted to methyl ester 5 [11], which was reacted with several boron acids under SuzukiMiyaura coupling conditions to give the corresponding 5-substituted product 6-8 in good yields [12]. The 5-substituted tropolones 9-11 were synthesized by the treatment of the corresponding methoxy compounds 6-8 with boron tribromide. All of the newly synthesized compounds were fully characterized by ${ }^{1} \mathrm{H}-\mathrm{NMR}$, IR, and elemental analyses. Table 1 shows the stretching vibrations of the carbonyl $\left(\mathrm{v}_{\mathrm{C}=\mathrm{O}}\right)$ groups of selected compounds, which were taken from their IR spectra, as well as their $\mathrm{pKa}$ values and partition coefficients.

In all cases, the stretching vibrations appeared around $1610 \mathrm{~cm}^{-1}$. Carbonyl compounds generally absorb in the region of $1700 \mathrm{~cm}^{-1}$, and the lower values observed in these compounds were attributed to the weakening of the double-bond character of their carbonyl group, because it formed an intramolecular hydrogen bond with the 2-hydroxyl group. This intramolecular hydrogen bond was clearly shown in the ${ }^{1} \mathrm{H}$-NMR spectra of the compounds, where the hydrogen of the hydroxyl group had been shifted to a much lower field than expected to appear as two peaks in the aromatic region. These data therefore suggested that 5-substituted tropolones are effectively 


\begin{tabular}{|l|c|c|c|c|}
\hline Compound & $\mathrm{R}$ & $\begin{array}{c}\mathrm{VC}=O \\
\left(\mathrm{~cm}^{-1}\right)\end{array}$ & $\mathrm{pKa}$ & $\log P_{\mathrm{o} / \mathrm{w}}$ \\
\hline 1 & $\mathrm{NO}_{2}$ & 1613 & 3.8 & $-\infty$ \\
\hline 4 & $\mathrm{I}$ & 1610 & 5.7 & -0.62 \\
\hline 2 & $\mathrm{NO}$ & 1613 & 5.9 & $-\infty$ \\
\hline 10 & $p$-CH - -phenyl & 1612 & 5.9 & $+\infty$ \\
\hline 11 & Thienyl & 1613 & 6.0 & 1.09 \\
\hline 9 & Phenyl & 1618 & 6.2 & 0.54 \\
\hline 3 & $\mathrm{NH}_{2}$ & 1662 & 8.1 & $-\infty$ \\
\hline
\end{tabular}

Table 1. Wavenumber of the stretching vibration of the carbonyl group in the of synthesized 5-substituted tropolones by IR spectroscopy, as well as their pKa values and partition coefficients.
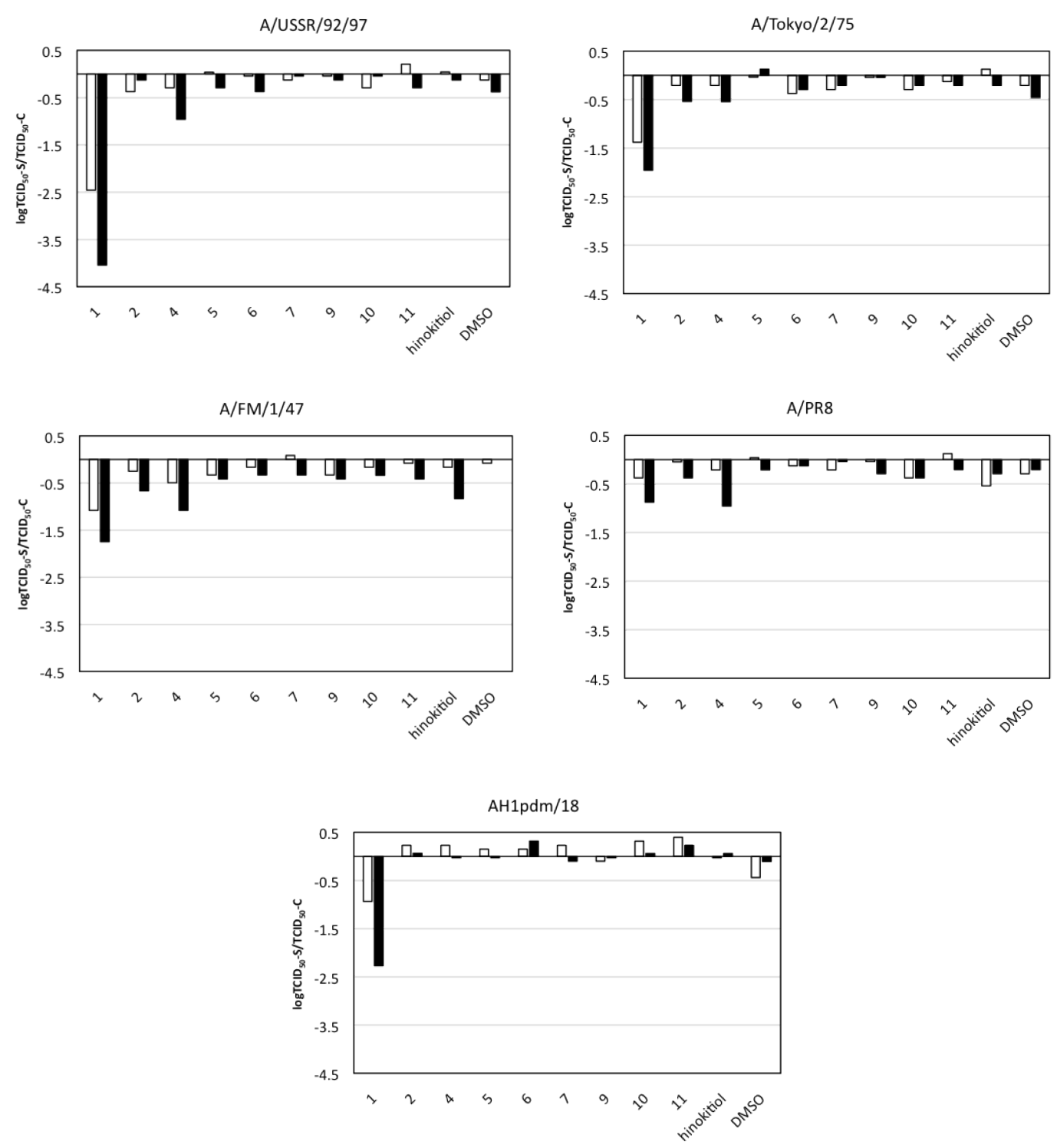

Figure 2. Effect of 5 -substituted tropolones on the virucidal activity. The activity shows the $\log \mathrm{TCID}_{50}-\mathrm{Se}_{\mathrm{TCID}}$ - $\mathrm{C}$ values. The contact times were $1 \mathrm{~min}$ (white bar) and $5 \mathrm{~min}$ (black bar). 
symmetrical compounds because of the hydrogen bond between these two groups. The $\pi$ electron of the carbonyl group would be delocalized, and the chemical shifts of the protons at C-4 and C- 6 of the tropolone ring tended to be shifted to a lower field following the incorporation of an electron-withdrawing group at C-5 of the tropolone ring, which resulted in a decrease in the electron density at these positions.

The electron donating ability of the functional group at C-5 had a significant impact on the $\mathrm{pKa}$ value of the 2-hydroxyl group as shown in Table 1. For example, the introduction of an electron-withdrawing nitro group at C-5 (i.e., compound 1) gave a $\mathrm{pKa}$ value of 3.8 for the 2 -hydroxyl group. This result indicated that compound 1 was effectively 1,000- and 10,000-fold more acidic than tropolone and hinokitiol, whose pKa values have been reported to be 6.7 and 7.2, respectively [13]. In contrast, the introduction of an electron-donating amino group at C-5 (i.e., compound 3) gave a $\mathrm{pKa}$ value of 8.1. The lower $\mathrm{pKa}$ of the hydroxyl group in nitro-containing compound 1 was attributed to the inductive withdrawal of electron density from the $\mathrm{O}$ atom of the hydroxyl group, which would effectively stabilize the anion formed by deprotonation. This anion would also be stabilized by the delocalization of its charge over the tropolone ring through a resonance effect. Based on these considerations, compound 1 could readily undergo a deprotonation reaction, as exemplified by its low $\mathrm{pKa}$ value. In contrast, the electron donating amino group in compound 3 would lead to the localization of the negative charge of the hydroxyl group on the $\mathrm{O}$ atom, which would effectively inhibit the deprotonation of the 2-hydroxyl group, leading to its observed higher $\mathrm{pK} a$ value.

\section{Virucidal activities of 5-substituted tropolones}

The virucidal activities of our newly synthesized 5-substituted tropolones were evaluated using a tissue culture infection doses $50\left(\mathrm{TICD}_{50}\right)$ assay. The activities of the samples were reported as TCID50/10 $\mu \mathrm{L}\left(\mathrm{TCID}_{50}-\mathrm{S}\right)$ divided by the value for control $\left(\mathrm{TCID}_{50}-\mathrm{C}\right)$. The results for five different types of influenza virus, including $\mathrm{A} /$ PR8, A/USSR/92/97, A/FM/1/47, AH1pdm/18 and A/Tokyo/2/75, are shown in Figure 2. Only compounds 1 and 4, bearing nitro and iodo groups at their C-5 positions, respectively, showed virucidal activities. Hinokitiol did not show virucidal activity towards any of the influenza viruses tested in the current study. The activity of compound 1 was higher than that of compound 4, and compound 1 showed its highest activity towards A/USSR/92/97. It is noteworthy that compounds 1 and 4 showed the lowest pKa values of the nine compounds tested in the current study. These results therefore suggest that the $\mathrm{pKa}$ value of the 2-hydroyl group, that is, the proton donating ability of the hydroxyl group, is an important factor in terms of defining the virucidal activity of tropolone compounds towards the influenza virus. A comparison of the 5-substituted compounds tested in the current study with a pKa of approximately 6 revealed that compound 4 was the only one of these compounds to show any virucidal activity. It had a halogen at its C-5 position and was the only one of the 2-hydroxyl-containing tropolone compounds tested in the current study with a $\mathrm{pKa}$ of approximately 6 to show any virucidal activity, suggesting that the presence of a halogen is important to the activity of these compounds. A comparison of compound 4 and 5, both of which have iodo group at its C-5 position, compound 5 did not show the virucidal activity. From these results, it was suggested that both of 2-hydroxyl and 5-iodo groups are necessary to show the activity.

In summary, nine 5-substituted tropolones have been synthesized and evaluated in terms of their virucidal activity towards five different types of influenza virus. Only 5-substituted tropolones bearing a nitro or iodo group exhibited any virucidal activity. These results suggest that the $\mathrm{pKa}$ value of the 2-hydroxyl group is an important factor in determining the virucidal activity of the tropolone derivatives, as well as the hydrophilic properties of the compounds.

\section{Conflicts of interest}

The authors declare no conflicts of interest associated with this manuscript.

\section{Supplementary data}

Supplementary data (general procedures and analytical data) associated with this article can be found online.

\section{References}

1. Uchida N, Ohyama K, Toyoda H. Current and Future Anti-Influenza Virus Drugs. The Open Antimicrob Agents J. 2010; 2:34-48.

2. Nozoe T. Über die Farbstoffe im Holzteile des "HINOKI"-Baumes. I. Hinokitin und hinokitiol. Bull Chem Soc Jpn 1936; 11:295-198.

3. Trust TJ, Coombs RW. Antibacterial activity of $\beta$-thujaplicin. Can J Microbiol 1973; 19:1341-1346.

4. Watanabe T, Ogasawara A, Mikami T, et al. Hyphal formation of Candida albicans is controlled by electron transfer system. Biochem Biophys Res Commun 2006; 348:206-211.

5. Shih $\mathrm{YH}, \mathrm{Chang}, \mathrm{KW}$, Hisa SM, et al. In vitro antimicrobial and anticancer potential of hinokitiol against oral pathogens and oral cancer cell lines. Microbiological research 2013, 168, 254.

6. Li LH, Wu P, Lee JY, et al. Hinokitiol Induces DNA Damage and Autophagy followed by Cell Cycle Arrest and Senescence in Gefitinib-Resistant Lung Adenocarcinoma Cells. Plos One 2014; 9: e104203.

7. Morita Y, Sakagami Y, Okabe T, et al. The Mechanism of the Bactericidal Activity of Hinokitiol. Biocontrol Sci 2017; 12: 101-110.

8. Adachi Y, Yoshida J, Kodera Y, et al. A new insulin-mimetic bis(allixinato) zinc(II) complex: structure-activity relationship of zinc(II) complexes. J Biol Inorg Chem 2014; 9: 885-893.

9. Potenziano J, Spitale R, Janik ME. Improved and Highly Versatile Synthesis of 5-Aryltropones. Synth Commun 2005; 35: 2005-2016.

10. Ito A, Muratake H, Shudo K. On the Structure of "5-Nitrosotropolone". J Org Chem 2009; 74:1275-1281.

11. Seganish WM, Handy CJ, DeShong P. Efforts Directed toward the Synthesis of Colchicine: Application of Palladium-Catalyzed Siloxane Cross-Coupling Methodology. J Org Chem 2005; 70:8948-8955.

12. Piettre SR, Andre'C, Chanal MC, et al. Monoaryl- and Bisaryldihydroxytropolones as Potent Inhibitors of Inositol Monophosphatase. J Med Chem 1997; 40:4208-4221

13. Doering WVE, Knox LH. Tropolone. J Am Chem Soc 1951; 73:828-838.

14. Yui N, Nozoe T (1951). Science Rep. of Tohoku University, Ser, 1 35: 83. 